\title{
RELAÇÕES FLORÍSTICO-GEOGRÁFICAS NA ESTRUTURA DE UMA FLORESTA NA REGIÃO CENTRAL DO RIO GRANDE DO SUL, BRASIL
}

\author{
Rafael Barbizan Sühs ${ }^{1}$, Jair Putzke ${ }^{2}$, Jean Carlos Budke ${ }^{3}$ \\ ${ }^{1}$ Bacharel em Ciências Biológicas, UNISC, Santa Cruz do Sul, RS, Brasil - rbsuhs@ gmail.com \\ ${ }^{2}$ Biólogo, Dr., Depto. Biologia e Farmácia, UNISC, Santa Cruz do Sul, RS, Brasil - jair@ unisc.br \\ ${ }^{3}$ Biólogo, Dr., Depto. Ciências Biológicas, URI, Campus de Erechim, RS, Brasil - jean@uricer.edu.br
}

Recebido para publicação: 21/08/2009 - Aceito para publicação: 16/12/2009

\begin{abstract}
Resumo
O presente estudo verificou a influência dos contingentes florísticos sobre a composição e estrutura de uma sinúsia florestal na região central do Rio Grande do Sul, Brasil. O estudo foi realizado no município de Sinimbu, em uma floresta decidual, onde foram demarcadas 100 unidades amostrais contíguas, totalizando 1 ha. Todos os indivíduos com diâmetro à altura do peito $\geq 4,8 \mathrm{~cm}$ foram amostrados e identificados. As famílias com maior riqueza específica foram Myrtaceae (18), Fabaceae (16) e Euphorbiaceae (10). A maior parte das espécies e dos indivíduos amostrados é de ampla distribuição geográfica. Uma elevada proporção das espécies pertenceu ao contingente das florestas estacionais, entretanto, este apresentou baixo número de indivíduos. O contingente Atlântico apresentou, além de um baixo número de indivíduos, também baixa riqueza. A comunidade apresentou três estratos de altura, todos com predominância de plantas de ampla distribuição geográfica.

Palavras-chave: Fitogeografia; flora; unidade de conservação; vale do Rio Pardo.
\end{abstract}

\begin{abstract}
Floristic-geographic relationships in a forest structure at the central region of the State of Rio Grande do Sul, Brazil. This work studied the influence of floristic contingents on the composition and structure of a forest in the central region of the State of Rio Grande do Sul, Brazil. The study was carried out in the County of Sinimbu, in a deciduous forest, where 100 contiguous sampling units that totaled 1 ha were established. All individuals with $\mathrm{DBH} \geq 4,8 \mathrm{~cm}$ were identified and measured. The richest families were Myrtaceae (18), Fabaceae (16) and Euphorbiaceae with 10 species. Most of the species surveyed are widely distributed across southern Brazilian vegetation formations. A large proportion of species belonged to the western seasonal contingent, although it has had contributed with few individuals. The eastern wet contingent presented both, low percentage of species and individuals. The community showed three height layers, all of them showing dominance of wide geographical distribution species.
\end{abstract}

Keywords: Phytogeography; flora; conservation unit; Rio Pardo valley.

\section{INTRODUÇÃO}

Na região Sul, a expansão das florestas tropicais e da Floresta com Araucária ocorreu somente no Holoceno, após o término da última era glacial e o consequente aumento da umidade (BEHLING, 1995; 1998). Anteriormente (Pleistoceno), no Sul e Sudeste do Brasil, as florestas tropicais, subtropicais e cerrados existentes foram muitas vezes substituídas por campos e algumas florestas subtropicais de galeria nos períodos pré e glacial (BEHLING, 2002). Somente a partir de 6.000 anos antes do presente (A.P.) é que as características geográficas gerais do território sul-rio-grandense se apresentam com uma fisionomia semelhante à atual (KERN, 1991).

Os trabalhos pioneiros de caracterização fitogeográfica do Rio Grande do Sul feitos por Rambo (1956; 1961) demonstraram que os dois únicos corredores de migração de espécies tropicais existentes no Rio Grande do Sul ocorrem pelo oeste (bacias dos rios Paraná e Uruguai) e pelo leste (Atlântico). Esses corredores se interpenetram nas encostas e base do Planalto Sul-Brasileiro, sendo essas áreas privilegiadas para o desenvolvimento de estudos ecológicos e fitogeográficos (JARENKOW; WAECHTER, 2001). Por 
outro lado, no Planalto Sul-Brasileiro, além de áreas de campo, distribui-se também a Floresta com Araucária (Floresta Ombrófila Mista), a qual possui influências florísticas com elementos não-tropicais provenientes dos Andes, da região austral-antártica e das montanhas do Brasil central, além de espécies tropicais (RAMBO, 1951; 1953; 1956). De acordo com Rambo (1953), essa flora típica não se desenvolveria em um clima tropical e, portanto, já ocupava o território do Rio Grande do Sul antes da chegada das espécies tropicais. Isso veio a ser corroborado em trabalhos paleoclimáticos recentes, realizados na região Sul e Sudeste do Brasil por Behling (1995; 1998; 2002) e Behling et al. (2001; 2004; 2005).

Em trabalho recente, Waechter (2002) relatou que os elementos florísticos do Rio Grande do Sul refletem padrões amplos e disjuntos com outros reinos florísticos. O mesmo autor separou a flora nativa do Rio Grande do Sul em elementos tropicais e temperados (subtropicais) e um de caráter cosmopolita, os quais, relacionados a uma série de fatores, refletem-se nos diversificados tipos florestais ocorrentes no Estado. Jarenkow; Waechter (2001) foram os primeiros a unir dados fitossociológicos às características fitogeográficas das espécies, ao estudarem um remanescente florestal na região central do Rio Grande do Sul.

Trabalhos estruturais recentes realizados por Jarenkow (1994), Jarenkow; Waechter (2001), Budke et al. (2004); Lindenmaier; Budke (2006) e Bergamin; Mondin (2006) estabeleceram relações entre a estrutura das florestas do Rio Grande do Sul e aspectos fitogeográficos. Entretanto pouco se sabe sobre a estrutura da vegetação das encostas erodidas da Serra Geral no Rio Grande do Sul, uma vez que o difícil acesso e a declividade dificultam a realização de trabalhos. Além disso, a influência dos contingentes florísticos sobre a estrutura destas florestas também é pouco conhecida.

Nessa perspectiva, objetivou-se com o presente estudo verificar a influência dos diferentes contingentes florísticos na composição e estrutura de uma sinúsia florestal localizada na porção meridional do Planalto Sul-Brasileiro.

\section{MATERIAL E MÉTODOS}

\section{Área do estudo}

O estudo foi realizado na Reserva Particular do Patrimônio Natural (RPPN) da Universidade de Santa Cruz do Sul, a qual está entre as maiores unidades de conservação (UC) dessa categoria no estado do Rio Grande do Sul (localização e altitude da sede da reserva em relação ao nível do mar: 29 23 '50' S - 52 32 '41' O; 375 m), possuindo 221,39 ha (Figura 1). Localiza-se no município de Sinimbu e constitui um importante remanescente para a região. Teve sua criação no ano de 2009 , através da Portaria $\mathrm{n}^{\circ} 16$, de 18 de março de 2009 (BRASIL, 2009), porém as atividades de pesquisa na área tiveram início já no ano de 2006. O uso dos recursos naturais por antigos moradores do local fez com que boa parte da vegetação nativa fosse removida para diversos fins, em especial para a criação de caprinos, agricultura e secagem de fumo. Concomitantemente, houve a introdução de espécies exóticas, para o fornecimento de madeira. As áreas visivelmente menos impactadas encontram-se nas encostas de morros, as quais dificultam a realização de atividades agro-silvi-pastoris, devido às condições topográficas. Esse dado pode ser aplicado a grande parte das encostas da Serra Geral no Rio Grande do Sul.

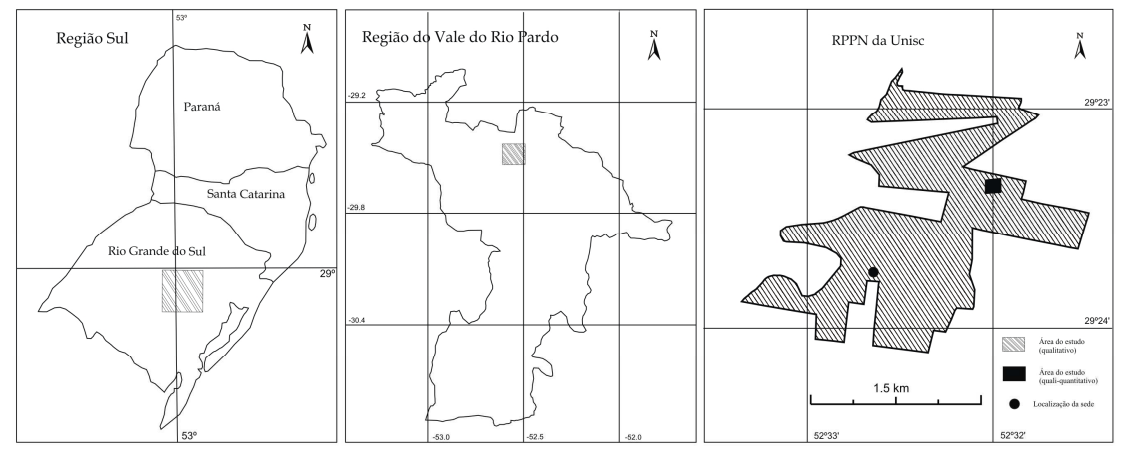

Figura 1. Localização da Reserva Particular do Patrimônio Natural da UNISC, região central do Rio Grande do Sul, Brasil.

Figure 1. Location of the Private Reserve of Natural Heritage from UNISC, central Rio Grande do Sul State, Brazil. 


\section{Clima}

Segundo a classificação de Maluf (2000), o clima da região é do tipo STSBv (subtropical, subúmido, com períodos de seca no verão). A temperatura média anual é de $19,3{ }^{\circ} \mathrm{C}$ e a precipitação média anual é de $1.547 \mathrm{~mm}$, registrados no município de Santa Cruz do Sul, estação de coleta de dados mais próxima (dados fornecidos pelo Laboratório de Meteorologia da Universidade de Santa Cruz do Sul).

\section{Solos e relevo}

Os solos dessa região pertencem a uma associação entre os solos das unidades de mapeamento Ciríaco e Charrua (STRECK et al., 2002), onde a unidade Ciríaco é tratada como "Chernossolo Argilúvico férrico típico" e a unidade Charrua como "Neossolo Litólico eutrófico chernossólico", na classificação proposta por EMBRAPA (1999).

Em geral, o relevo da Reserva Particular do Patrimônio Natural da UNISC apresenta altitudes mínimas em torno de $150 \mathrm{~m}$ ao leste, junto ao leito do rio Pardinho, e as maiores no topo de morros, atingindo em torno de $650 \mathrm{~m}$.

\section{Procedimento amostral e análise dos dados}

A composição florística e estrutural foi verificada em uma área de 1 ha, onde aplicou-se o método de parcelas (MUELLER-DOMBOIS; ELLENBERG, 1974). Instalou-se uma parcela de $100 \mathrm{~m} \mathrm{x}$ $100 \mathrm{~m}$ (1 ha) em uma área de encosta declivosa, a qual foi subdividida em 100 unidades amostrais

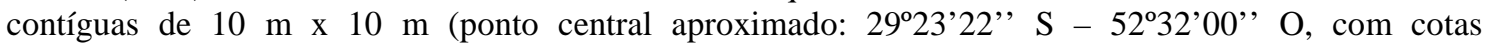
altimétricas variando de $460 \mathrm{~m}$ a $570 \mathrm{~m}$ ). Determinou-se esse local por apresentar, visualmente, vegetação menos alterada por atividades antrópicas recentes, quando comparada a outros lugares dentro da UC. Todos os indivíduos que apresentaram diâmetro à altura do peito (DAP) maior ou igual a $4,8 \mathrm{~cm}$ foram identificados e tiveram altura estimada por comparação a uma vara de altura conhecida. Espécimes não identificados in loco foram coletados e registrados no herbário da Universidade de Santa Cruz do Sul (HCB), para posterior identificação.

A identificação dos espécimes foi feita através de literatura especializada, consultas a especialistas e por comparação a outras exsicatas. A classificação de famílias das angiospermas foi delimitada de acordo com a proposta de Angiosperm Phylogeny Group - APG II (2003), e, para samambaias arborescentes, utilizou-se o sistema de Smith et al. (2006). A nomenclatura das espécies seguiu principalmente Sobral et al. (2006).

Com o intuito de aumentar os dados sobre a flora local, utilizaram-se dados obtidos com o método de caminhamento (FILGUEIRAS et al., 1994). Entre abril de 2006 e abril de 2009, a área (221,39 ha) foi percorrida de forma esporádica e os indivíduos que apresentaram diâmetro à altura do peito (DAP) maior ou igual a $4,8 \mathrm{~cm}$ foram fotografados in loco e identificados. Sempre que possível, os indivíduos foram coletados e armazenados no herbário da Universidade de Santa Cruz do Sul (HCB).

Verificaram-se os contingentes florísticos das espécies amostradas com base principalmente nos trabalhos de Jarenkow (1994), Jarenkow; Waechter (2001), Budke et al. (2004) e Lindenmaier; Budke (2006). As espécies foram divididas em três categorias (JARENKOW; WAECHTER, 2001):

- espécie de ampla distribuição (EAD);

- espécie característica das bacias dos rios Paraná e Uruguai (BPU);

- espécie característica do contingente Atlântico (ATL).

As espécies características da região da Floresta Ombrófila Mista e as espécies exóticas foram enquadradas em outras duas categorias:

- espécie característica dos pinhais (PIN);

- espécie exótica (EXT).

Para o enquadramento das espécies nessas categorias, diversos trabalhos foram consultados, em especial Rambo (1951; 1953; 1956), Jarenkow (1994), Jarenkow; Waechter (2001), Budke et al. (2004) e Lindenmaier; Budke (2006). Calculou-se a média de altura de cada espécie juntamente com seu respectivo desvio padrão. O coeficiente de variação dos valores de desvio padrão também foi calculado, uma vez que esse cálculo permite comparar os valores obtidos. Elaborou-se um diagrama de classes de altura para facilitar a interpretação dos resultados. 


\section{RESULTADOS E DISCUSSÃO}

\section{Levantamento florístico}

Somando ambos os levantamentos (caminhamento e parcelas), encontraram-se 160 espécies distribuídas em 123 gêneros e 54 famílias. Quatro morfotipos foram identificados somente até nível de gênero, um ao nível de família e os demais ao nível de espécie (Tabela 1). A família que apresentou maior riqueza específica foi Myrtaceae, com 18 espécies, seguida por Fabaceae, com 16, e Euphorbiaceae, com 10.

Tabela 1. Famílias, espécies, números de coletas e parâmetros analisados de árvores encontradas na Reserva Particular do Patrimônio Natural da UNISC, Sinimbu, RS, Brasil, ordenadas por valor decrescente de número de indivíduos por família.

Table 1. Families, species, collector number (voucher) and analyzed parameters from trees surveyed in the Private Reserve of Natural Heritage from UNISC, Sinimbu, RS, Brazil, ordered by decreased values of individuals per family.

\begin{tabular}{|c|c|c|c|c|c|c|c|}
\hline Família/espécie & $\mathbf{N}$ & $\mathbf{C F}$ & $\mathbf{N i}$ & $\mathbf{I}$ & HM & $\mathbf{s}$ & $\mathrm{CV}$ \\
\hline SALICACEAE & & & 175 & 17,1 & & & \\
\hline Banara parviflora (A. Gray) Benth. & 080492 & EAD & 18 & 1,8 & 9,1 & 1,9 & 20,9 \\
\hline Banara tomentosa Clos & 070429 & BPU & 9 & 0,9 & 7,9 & 2,0 & 24,9 \\
\hline Casearia decandra Jacq. & 080125 & BPU & 5 & 0,5 & 8,6 & 1,7 & 19,5 \\
\hline Casearia sylvestris $\mathrm{Sw}$. & 080606 & EAD & 127 & 12,4 & 8,2 & 1,6 & 18,7 \\
\hline Salix humboldtiana Willd. & 080628 & EAD & & & & & \\
\hline Xylosma pseudosalzmannii Sleumer & 070188 & BPU & 16 & 1,6 & 7,6 & 1,3 & 16,7 \\
\hline LAURACEAE & & & 127 & 12,4 & & & \\
\hline Aiouea saligna Meisn. & 090044 & ATL & 6 & 0,6 & 10,5 & 4,6 & 42,2 \\
\hline Nectandra lanceolata Nees & 070418 & $\mathrm{BPU}$ & 2 & 0,2 & 11,0 & 0,0 & 0,0 \\
\hline Nectandra megapotamica (Spreng.) Mez & 070577 & EAD & 106 & 10,4 & 13,2 & 2,7 & 20,5 \\
\hline Ocotea puberula (Rich.) Nees & 060075 & EAD & 12 & 1,2 & 13,3 & 1,8 & 13,7 \\
\hline Ocotea pulchella (Nees) Mez & 080650 & EAD & 1 & 0,1 & 12,0 & & \\
\hline Persea americana Mill. & $\mathrm{NC}$ & EXT & & & & & \\
\hline EUPHORBIACEAE & & & 126 & 12,3 & & & \\
\hline Alchornea triplinervia (Spreng.) Müll. Arg. & 070578 & EAD & 72 & 7,0 & 12,6 & 2,5 & 19,7 \\
\hline Gymnanthes concolor Spreng. & 060076 & EAD & 8 & 0,8 & 5,0 & 0,9 & 18,5 \\
\hline Manihot grahamii Hook. & $\mathrm{NC}$ & EAD & & & & & \\
\hline Pachystroma longifolium (Ness) I.M. Johnst. & 080995 & ATL & & & & & \\
\hline Sapium glandulosum (L.) Morong. & 080617 & EAD & 1 & 0,1 & 9,0 & & \\
\hline Sebastiania brasiliensis Spreng. & $\mathrm{NC}$ & EAD & 1 & 0,1 & 4,0 & & \\
\hline Sebastiania commersoniana (Baill.) L. B. Sm. \& Downs & 070683 & EAD & 15 & 1,5 & 8,4 & 1,5 & 17,9 \\
\hline Stillingia oppositifolia Baill. Ex Müll. Arg. & 070220 & BPU & & & & & \\
\hline Tetrorchidium rubrivenium Poepp. \& Endl. & $\mathrm{NC}$ & EAD & 29 & 2,8 & 14,0 & 3,4 & 24,5 \\
\hline SAPINDACEAE & & & 118 & 11,5 & & & \\
\hline $\begin{array}{l}\text { Allophylus edulis (A.St.-Hil., Cambess \& A. } \\
\text { Juss.) Radlk. }\end{array}$ & 080291 & EAD & 51 & 5,0 & 8,0 & 1,7 & 21,5 \\
\hline Allophylus guaraniticus (A.St.-Hil.) Radlk. & 060071 & BPU & & & & & \\
\hline Cupania vernalis Cambess. & 080485 & EAD & 67 & 6,5 & 9,5 & 2,8 & 29,0 \\
\hline Matayba elaeagnoides Radlk. & 080618 & EAD & & & & & \\
\hline MORACEAE & & & 104 & 10,2 & & & \\
\hline Ficus cestrifolia Schott & $\mathrm{NC}$ & ATL & & & & & \\
\hline Ficus luschnathiana (Miq.) Miq. & 070432 & EAD & 10 & 1,0 & 12,2 & 2,4 & 19,6 \\
\hline Morus nigra $\mathrm{L}$. & 080128 & EXT & & & & & \\
\hline $\begin{array}{l}\text { Sorocea bonplandii (Baill.) W.C. Burger, } \\
\text { Lanjouw \& Boer }\end{array}$ & 070196 & EAD & 94 & 9,2 & 6,1 & 1,1 & 17,6 \\
\hline MELIACEAE & & & 82 & 8,0 & & & \\
\hline Cabralea canjerana (Vell.) Mart. & 080660 & EAD & 40 & 3,9 & 10,3 & 2,5 & 24,4 \\
\hline Cedrela fissilis Vell. & 080659 & EAD & 16 & 1,6 & 12,3 & 3,6 & 28,9 \\
\hline Trichilia claussenii C.DC. & 070440 & $\mathrm{BPU}$ & 23 & 2,2 & 7,4 & 1,2 & 16,7 \\
\hline
\end{tabular}




\begin{tabular}{|c|c|c|c|c|c|c|c|}
\hline Trichilia elegans A. Juss. & 080469 & BPU & 3 & 0,3 & 5,0 & 1,0 & 20,0 \\
\hline MYRSINACEAE & & & 80 & 7,8 & & & \\
\hline Myrsine coriacea (Sw.) R.Br. & 070575 & EAD & 1 & 0,1 & 13,0 & & \\
\hline Myrsine guianensis (Aubl.) Kuntze & 090038 & EAD & 46 & 4,5 & 8,8 & 1,9 & 21,2 \\
\hline Myrsine laetevirens (Mez) Arechav. & 070685 & BPU & & & & & \\
\hline Myrsine umbellata Mart. & 070189 & EAD & 33 & 3,2 & 9,6 & 1,7 & 17,9 \\
\hline FABACEAE & & & 52 & 5,1 & & & \\
\hline Albizia edwallii (Hoehne) Barneby \& J.Grimes & 080613 & BPU & 2 & 0,2 & 12,0 & 5,7 & 47,1 \\
\hline Bauhinia forficata Link & 070567 & EAD & & & & & \\
\hline Calliandra foliolosa Benth. & 080471 & BPU & & & & & \\
\hline Dalbergia frutescens (Vell.) Britton & 070419 & BPU & 3 & 0,3 & 8,3 & 0,6 & 6,9 \\
\hline Enterolobium contorstisiliquum (Vell.) Morong & 090042 & BPU & 1 & 0,1 & 13,0 & & \\
\hline Erythrina falcata Benth. & $\mathrm{NC}$ & BPU & & & & & \\
\hline Inga marginata Willd. & 070212 & EAD & 1 & 0,1 & 8,0 & & \\
\hline Inga vera Willd. & 070219 & BPU & 3 & 0,3 & 14,3 & 1,2 & 8,1 \\
\hline Inga virescens Benth. & 070692 & BPU & & & & & \\
\hline Lonchocarpus nitidus (Vogel) Benth. & 070434 & BPU & 5 & 0,5 & 10,8 & 3,1 & 28,8 \\
\hline Machaerium paraguariense Hassl. & 060072 & BPU & 19 & 1,9 & 9,2 & 2,2 & 23,9 \\
\hline Mimosa bimucronata (DC.) Kuntze & 070218 & EAD & & & & & \\
\hline Mimosa scabrella Benth. & 080092 & BPU & & & & & \\
\hline Myrocarpus frondosus Allemão & 070424 & BPU & 6 & 0,6 & 9,8 & 2,3 & 23,6 \\
\hline Parapiptadenia rigida (Benth.) Brenan & 080610 & BPU & 12 & 1,2 & 9,3 & 2,5 & 26,4 \\
\hline Senna pendula (Willd.) H.S. Irwin \& Barneby & 080079 & BPU & & & & & \\
\hline EBENACEAE & & & 21 & 2,1 & & & \\
\hline Diospyros inconstans Jacq. & 080619 & BPU & 21 & 2,1 & 7,0 & 2,1 & 30,0 \\
\hline Diospyros kaki L. & 090048 & EXT & & & & & \\
\hline MONIMIACEAE & & & 20 & 2,0 & & & \\
\hline Hennecartia omphalandra J. Poiss. & 080627 & BPU & 5 & 0,5 & 7,0 & 1,6 & 22,6 \\
\hline Mollinedia schottiana (Spreng.) Perkins & 070187 & ATL & 15 & 1,5 & 4,7 & 0,8 & 17,5 \\
\hline CUNONIACEAE & & & 14 & 1,4 & & & \\
\hline Lamanonia ternata Vell. & 070591 & EAD & 14 & 1,4 & 10,5 & 1,9 & 18,2 \\
\hline MELASTOMATACEAE & & & 14 & 1,4 & & & \\
\hline Leandra regnellii (Triana) Cogn. & 070594 & $\mathrm{EAD}$ & & & & & \\
\hline Miconia pusilliflora (DC.) Naudin & 070581 & EAD & 14 & 1,4 & 8,9 & 1,8 & 20,4 \\
\hline Miconia sp. & 070695 & - & & & & & \\
\hline SAPOTACEAE & & & 13 & 1,3 & & & \\
\hline $\begin{array}{l}\text { Chrysophyllum gonocarpum (Mart. \& Eichler) } \\
\text { Engl. }\end{array}$ & 080135 & BPU & 3 & 0,3 & 9,0 & 1,7 & 19,2 \\
\hline $\begin{array}{l}\text { Chrysophyllum marginatum (Hook. \& Arn.) } \\
\text { Radlk. }\end{array}$ & 070436 & BPU & 10 & 1,0 & 9,3 & 1,5 & 16,1 \\
\hline RUTACEAE & & & 11 & 1,1 & & & \\
\hline Citrus x limon (L.) Burm. & 080616 & EXT & 1 & 0,1 & 5,0 & & \\
\hline Citrus reticulata Blanco & 080468 & EXT & & & & & \\
\hline Citrus $x$ sinensis $(\mathrm{L}$.$) Osbeck$ & $\mathrm{NC}$ & EXT & & & & & \\
\hline Helietta apiculata Benth. & 070582 & BPU & & & & & \\
\hline Zanthoxylum caribaeum Lam. & 070680 & EAD & 10 & 1,0 & 8,6 & 1,4 & 16,6 \\
\hline Zanthoxylum fagara (L.) Sarg. & 080620 & BPU & & & & & \\
\hline Zanthoxylum rhoifolium Lam. & 070195 & $\mathrm{EAD}$ & & & & & \\
\hline BORAGINACEAE & & & 11 & 1,1 & & & \\
\hline Cordia americana (L.) Gottschiling \& J. E. Mill. & 070576 & BPU & 1 & 0,1 & 6,0 & & \\
\hline Cordia ecalyculata Vell. & 080285 & BPU & 3 & 0,3 & 6,7 & 0,6 & 8,7 \\
\hline Cordia trichotoma (Vell.) Arrab. ex Steud. & 080140 & $\mathrm{EAD}$ & 7 & 0,7 & 9,1 & 2,3 & 25,6 \\
\hline MALVACEAE & & & 9 & 0,9 & & & \\
\hline Luehea divaricata Mart. \& Zucc. & 080611 & EAD & 9 & 0,9 & 9,4 & 2,7 & 28,6 \\
\hline MYRTACEAE & & & 6 & 0,6 & & & \\
\hline
\end{tabular}

FLORESTA, Curitiba, PR, v. 40, n. 3, p. 635-646, jul./set. 2010. Sühs, R. B.; Putzke, J.; Budke, J. C. 


\begin{tabular}{|c|c|c|c|c|c|c|c|}
\hline Acca sellowiana $(\mathrm{O}$. Berg) Burret & 080657 & BPU & & & & & \\
\hline Blepharocalyx salicifolius (Kunth) O. Berg & 090039 & EAD & & & & & \\
\hline Calyptranthes concinna DC. & 070579 & BPU & & & & & \\
\hline Calyptranthes grandifolia O. Berg & 090051 & ATL & & & & & \\
\hline Campomanesia guazumifolia (Cambess.) O. Berg & $\mathrm{NC}$ & EAD & & & & & \\
\hline Campomanesia xanthocarpa O.Berg & 070678 & $\mathrm{EAD}$ & & & & & \\
\hline Eucalyptus sp. & 090053 & EXT & & & & & \\
\hline Eugenia hiemalis Cambess. & 070681 & ATL & & & & & \\
\hline Eugenia involucrata DC. & 080519 & BPU & 2 & 0,2 & 7,5 & 2,1 & 28,3 \\
\hline Eugenia rostrifolia D.Legrand & 080490 & BPU & 2 & 0,2 & 7,5 & 0,7 & 9,4 \\
\hline Eugenia uniflora $\mathrm{L}$. & 070677 & EAD & 2 & 0,2 & 7,0 & 1,4 & 20,2 \\
\hline $\begin{array}{l}\text { Myrceugenia glaucescens (Cambess.) D.Legrand } \\
\text { \& Kausel }\end{array}$ & 090036 & BPU & & & & & \\
\hline Myrcianthes gigantea (D.Legrand) D.Legrand & 090054 & BPU & & & & & \\
\hline Myrcianthes pungens (O.Berg) D.Legrand & 070200 & BPU & & & & & \\
\hline Myrciaria sp. & $\mathrm{NC}$ & - & & & & & \\
\hline Myrrhinium atropurpureum Schott & 070194 & BPU & & & & & \\
\hline Psidium cattleianum Sabine & $\mathrm{NC}$ & $\mathrm{EAD}$ & & & & & \\
\hline não identificada & 090049 & - & & & & & \\
\hline CYATHEACEAE & & & 6 & 0,6 & & & \\
\hline Alsophila setosa Kaulf. & 080651 & EAD & 6 & 0,6 & 4,2 & 1,8 & 44,0 \\
\hline ANNONACEAE & & & 5 & 0,5 & & & \\
\hline Annona sp. & 080284 & & & & & & \\
\hline Annona neosalicifolia (Schltdl.) H.Rainer & 080282 & BPU & & & & & \\
\hline Annona sylvatica A. St.-Hil. & 090022 & EAD & 5 & 0,5 & 6,4 & 1,5 & 23,7 \\
\hline NYCTAGINACEAE & & & 5 & 0,5 & & & \\
\hline Pisonia zapallo Griseb. & 080493 & $\mathrm{EAD}$ & 5 & 0,5 & 8,0 & 2,1 & 26,5 \\
\hline SIMAROUBACEAE & & & 5 & 0,5 & & & \\
\hline Picrasma crenata (Vell.) Engl. & 080131 & BPU & 5 & 0,5 & 9,0 & 2,7 & 30,4 \\
\hline LAMIACEAE & & & 6 & & & & \\
\hline Aegiphila brachiata Vell. & 080139 & ATL & 3 & 0,3 & & & \\
\hline Vitex megapotamica (Spreng.) Moldenke & 080631 & EAD & 3 & 0,3 & 8,3 & 3,5 & 42,1 \\
\hline \multicolumn{8}{|l|}{ RUBIACEAE } \\
\hline Cephalanthus glabratus (Spreng.) K. Schum. & $\mathrm{NC}$ & BPU & 3 & 0,3 & & & \\
\hline Chomelia obtusa Cham. \& Schltdl. & 070573 & EAD & & & & & \\
\hline Coutarea hexandra (Jacq.) K. Schum. & 070449 & BPU & 2 & 0,2 & 8,0 & 0,0 & 0,0 \\
\hline Guettarda uruguensis Cham. \& Schltdl. & 080975 & BPU & & & & & \\
\hline Randia ferox (Cham. \& Schltdl.) DC. & 070191 & $\mathrm{EAD}$ & 1 & 0,1 & 7,0 & & \\
\hline Rudgea parquioides (Cham.) Müll.Arg. & 080614 & $\mathrm{EAD}$ & & & & & \\
\hline ANACARDIACEAE & & & 2 & 0,2 & & & \\
\hline Lithraea brasiliensis Marchand & 080288 & BPU & 2 & 0,2 & 10,0 & 0,0 & 0,0 \\
\hline Schinus terebinthifolius Raddi & 080655 & EAD & & & & & \\
\hline PROTEACEAE & & & 2 & 0,2 & & & \\
\hline Roupala brasiliensis Klotzsch & 080287 & PIN & 2 & 0,2 & 8,5 & 0,7 & 8,3 \\
\hline RHAMNACEAE & & & 2 & 0,2 & & & \\
\hline Hovenia dulcis Thunb. & 080467 & EXT & 2 & 0,2 & 11,0 & 5,7 & 51,4 \\
\hline URTICACEAE & & & 2 & 0,2 & & & \\
\hline Boehmeria caudata Sw. & 070570 & EAD & & & & & \\
\hline Urera baccifera (L.) Gaudich. & $\mathrm{NC}$ & BPU & 2 & 0,2 & 5,5 & 0,7 & 12,9 \\
\hline ARALIACEAE & & & 1 & 0,1 & & & \\
\hline Schefflera morototoni (Aubl.) Maguire, Steyerm. \& Frondin & 070690 & BPU & 1 & 0,1 & 17,0 & & \\
\hline BIGNONIACEAE & & & 1 & 0,1 & & & \\
\hline Jacaranda micrantha Cham. & $\mathrm{NC}$ & BPU & & & & & \\
\hline Handroanthus albus (Cham.) Mattos & 080652 & BPU & & & & & \\
\hline Tecoma stans (L.) Kunth & 080607 & EXT & 1 & 0,1 & 9,0 & & \\
\hline
\end{tabular}




\begin{tabular}{|c|c|c|c|c|c|}
\hline CANNABACEAE & & & 1 & 0,1 & \\
\hline Celtis iguanaea (Jacq.) Sarg. & 060069 & BPU & & & \\
\hline Trema micrantha (L.) Blume & 080484 & EAD & 1 & 0,1 & 7,0 \\
\hline CARICACEAE & & & 1 & 0,1 & \\
\hline Vasconcella quercifolia A. St.-Hil. & 090046 & EAD & 1 & 0,1 & 8,0 \\
\hline ROSACEAE & & & 1 & 0,1 & \\
\hline Eriobotrya japonica (Thunb.) Lindl. & 090030 & EXT & & & \\
\hline Prunus myrtifolia (L.) Urb. & 090047 & EAD & 1 & 0,1 & 15,0 \\
\hline SOLANACEAE & & & 1 & 0,1 & \\
\hline Brugmansia suaveolens Bercht. \& Presl. & 080604 & EXT & & & \\
\hline Brunfelsia cuneifolia J. A. Schmidt & 070691 & BPU & & & \\
\hline Cestrum intermedium Sendtn. & 070674 & BPU & 1 & 0,1 & 8,0 \\
\hline Solanum mauritianum Scop. & 080605 & EAD & & & \\
\hline Solanum paranense Dusén & 080516 & BPU & & & \\
\hline Solanum pseudoquina A. St.-Hil. & 070417 & ATL & & & \\
\hline Solanum sanctaecatharinae Dunal & 070427 & EAD & & & \\
\hline Vassobia breviflora (Sendtn.) Hunz. & 080466 & EAD & & & \\
\hline \multicolumn{6}{|l|}{ ADOXACEAE } \\
\hline Sambucus australis Cham. \& Schltdl. & 070192 & EAD & & & \\
\hline \multicolumn{6}{|l|}{ AQUIFOLIACEAE } \\
\hline Ilex paraguariensis A. St.-Hil. & 080470 & EAD & & & \\
\hline \multicolumn{6}{|l|}{ ARAUCARIACEAE } \\
\hline Araucaria angustifolia (Bertol.) Kuntze & 080654 & PIN & & & \\
\hline \multicolumn{6}{|l|}{ ARECACEAE } \\
\hline Syagrus romanzoffiana (Cham.) Glassman & 080476 & $\mathrm{EAD}$ & & & \\
\hline \multicolumn{6}{|l|}{ ASTERACEAE } \\
\hline Baccharis semiserrata DC. & 080453 & EAD & & & \\
\hline Dasyphyllum spinescens (Less.) Cabrera & 070437 & BPU & & & \\
\hline Dasyphyllum tomentosum (Spreng.) Cabrera & 080632 & BPU & & & \\
\hline Eupatorium rufescens Lund ex DC. & 070210 & BPU & & & \\
\hline Gochnatia polymorpha (Less.) Cabrera & $\mathrm{NC}$ & EAD & & & \\
\hline Piptocarpha tomentosa Baker & 070689 & ATL & & & \\
\hline Vernonia discolor (Spreng.) Less. & 080094 & $\mathrm{EAD}$ & & & \\
\hline \multicolumn{6}{|l|}{ CACTACEAE } \\
\hline Cereus hildmannianus K. Schum. & $\mathrm{NC}$ & $\mathrm{EAD}$ & & & \\
\hline \multicolumn{6}{|l|}{ CARDIOPTERIDACEAE } \\
\hline Citronella paniculata (Mart.) R.A. Howard & 070216 & EAD & & & \\
\hline \multicolumn{6}{|l|}{ CELASTRACEAE } \\
\hline Maytenus aquifolia Mart. & 070441 & BPU & & & \\
\hline Schaefferia argentinensis Speg. & 080150 & BPU & & & \\
\hline \multicolumn{6}{|l|}{ DICKSONIACEAE } \\
\hline Dicksonia sellowiana Hook & $\mathrm{NC}$ & BPU & & & \\
\hline \multicolumn{6}{|l|}{ ELAEOCARPACEAE } \\
\hline Sloanea monosperma Vell. & 070207 & ATL & & & \\
\hline \multicolumn{6}{|l|}{ ERYTHROXYLACEAE } \\
\hline Erythroxylum argentinum O.E. Schulz & 070438 & EAD & & & \\
\hline Erythroxylum deciduum A. St.-Hil. & 060070 & EAD & & & \\
\hline \multicolumn{6}{|l|}{ ESCALLONIACEAE } \\
\hline Escallonia bifida Link \& Otto & 070208 & PIN & & & \\
\hline \multicolumn{6}{|l|}{ LOGANIACEAE } \\
\hline Strychnos brasiliensis (Spreng.) Mart. & 080609 & EAD & & & \\
\hline \multicolumn{6}{|l|}{ PHYLLANTHACEAE } \\
\hline $\begin{array}{l}\text { Phyllanthus sellowianus (Klotzsch) Müll. Arg. } \\
\text { PHYTOLACCACEAE }\end{array}$ & 080633 & $\mathrm{EAD}$ & & & \\
\hline
\end{tabular}

FLORESTA, Curitiba, PR, v. 40, n. 3, p. 635-646, jul./set. 2010. Sühs, R. B.; Putzke, J.; Budke, J. C. 


\begin{tabular}{|c|c|c|}
\hline Phytolacca dioica $\mathrm{L}$. & 080612 & EAD \\
\hline Seguieria aculeata Jacq. & 070201 & EAD \\
\hline \multicolumn{3}{|l|}{ PICRAMNIACEAE } \\
\hline Picramnia parvifolia Engl. & 080290 & ATL \\
\hline \multicolumn{3}{|l|}{ PIPERACEAE } \\
\hline Piper aduncum $\mathrm{L}$. & 070590 & EAD \\
\hline Piper amalago $\mathrm{L}$. & 080457 & $\mathrm{BPU}$ \\
\hline \multicolumn{3}{|l|}{ PODOCARPACEAE } \\
\hline Podocarpus lambertii Klotzsch ex Endl. & 080133 & PIN \\
\hline \multicolumn{3}{|l|}{ POLYGONACEAE } \\
\hline Ruprechtia laxiflora Meisn. & 070679 & $\mathrm{BPU}$ \\
\hline \multicolumn{3}{|l|}{ SABIACEAE } \\
\hline Meliosma sellowii Urb. & 080653 & ATL \\
\hline \multicolumn{3}{|l|}{ STYRACACEAE } \\
\hline Styrax leprosus Hook \& Arn. & 080489 & EAD \\
\hline
\end{tabular}

N: número de coleta; CF: característica fitogeográfica; Ni: Número de indivíduos; I: porcentagem de indivíduos amostrados (\%); HM: altura média (metros); s: desvio padrão (metros); CV: coeficiente de variação (\%). NC: não coletada; EAD: espécie de ampla distribuição; BPU: espécie das bacias dos rios Paraná e Uruguai; ATL: espécie do contingente Atlântico; PIN: espécie dos pinhais; EXT: espécie exótica.

Em relação aos contingentes florísticos e demais categorias, foram analisadas 156 espécies. Destas, 44\% são espécies de ampla distribuição, 38\% são provenientes das bacias dos Rios Paraná e Uruguai (oeste), $8 \%$ do contingente Atlântico (leste), 3\% são espécies características dos pinhais e 7\% exóticas.

Foram encontradas 11 espécies exóticas, das quais cinco são consideradas invasoras pelo governo do estado do Paraná (PARANÁ, 2007): Tecoma stans (L.) Kunth; Morus nigra L.; Hovenia dulcis Thunb.; Citrus x limon (L.) Burm. e Eriobotrya japonica (Thunb.) Lindl. Nesse aspecto, tem-se um dado preocupante para a área, uma vez que as espécies exóticas invasoras estão atualmente entre as maiores ameaças biológicas ao meio ambiente (IUCN, 2000). Essas espécies acarretam mudanças e alterações nas propriedades do solo e na ciclagem de nutrientes, nas cadeias tróficas, na estrutura, nas funções dos ecossistemas, na distribuição da biomassa, na taxa de decomposição, nos processos evolutivos e nas relações entre polinizadores, além de poder produzir híbridos com espécies nativas e assim eliminar genótipos naturais (IUCN, 2000; MACK et al. 2000; ZILLER; GALVÃO, 2003).

Foram encontradas 12 espécies ameaçadas de extinção de acordo com as listas Flora Ameaçada do Rio Grande do Sul (RIO GRANDE DO SUL, 2003), Flora Brasileira Ameaçada de Extinção (BIODIVERSITAS, 2005) e lista da União Internacional para a Conservação da Natureza (IUCN, 2008), apresentadas na tabela 2 .

Tabela 2. Espécies ameaçadas de extinção encontradas na RPPN da UNISC, Sinimbu, RS, Brasil, conforme as listas de Rio Grande do Sul (2003), IUCN (2008) e Biodiversitas (2005).

Table 2. Threatened species found in the RPPN from UNISC, Sinimbu, RS, Brazil, according to lists from Rio Grande do Sul (2003), IUCN (2008) and Biodiversitas (2005.

\begin{tabular}{llccc}
\hline Família & Espécie & RS & Brasil & IUCN \\
\hline ARAUCARIACEAE & Araucaria angustifolia (Bertol.) Kuntze & VU & EN & VU \\
ASTERACEAE & Gochnatia polymorpha (Less.) Cabrera & VU & - & - \\
CELASTRACEAE & Maytenus aquifolia Mart. & VU & - & - \\
DICKSONIACEAE & Dicksonia sellowiana Hook & VU & EN & - \\
FABACEAE & Albizia edwallii (Hoehne) Barneby \& J.Grimes & - & - & VU \\
& Myrocarpus frondosus Allemão & VU & - & DD \\
MELIACEAE & Cedrela fissilis Vell. & - & - & EN \\
MYRTACEAE & Myrcianthes pungens (O.Berg) D.Legrand & - & - & EN \\
PODOCARPACEAE & Podocarpus lambertii Klotzsch ex Endl. & - & - & DD \\
RUBIACEAE & Rudgea parquioides (Cham.) Müll.Arg. & - & EN/VU & - \\
PICRAMNIACEAE & Picramnia parvifolia Engl. & VU & - & - \\
SIMAROUBACEAE & Picrasma crenata (Vell.) Engl. & VU & - & - \\
\hline VU:
\end{tabular}

VU: vulnerável; EM: em perigo; DD: dados insuficientes. 


\section{Levantamento estrutural}

Registraram-se 1.063 indivíduos, sendo 1.024 vivos e 39 mortos ainda em pé (4\% do total). Foram amostradas 69 espécies pertencentes a 33 famílias. A família de maior riqueza foi Fabaceae, com nove espécies, seguida por Euphorbiaceae, com seis. Em relação às famílias com maior abundância, Salicaceae esteve à frente, com mais de $17 \%$ do total de indivíduos amostrados, seguida por Lauraceae, Euphorbiaceae e Sapindaceae, cada uma com pouco mais de $11 \%$ do total.

Com relação à altura, $52 \%$ dos indivíduos apresentaram altura entre $6 \mathrm{~m}$ e $9 \mathrm{~m}$, indicando que grande parte dos espécimes possui porte médio ou são arvoretas do sub-bosque (Figura 2).

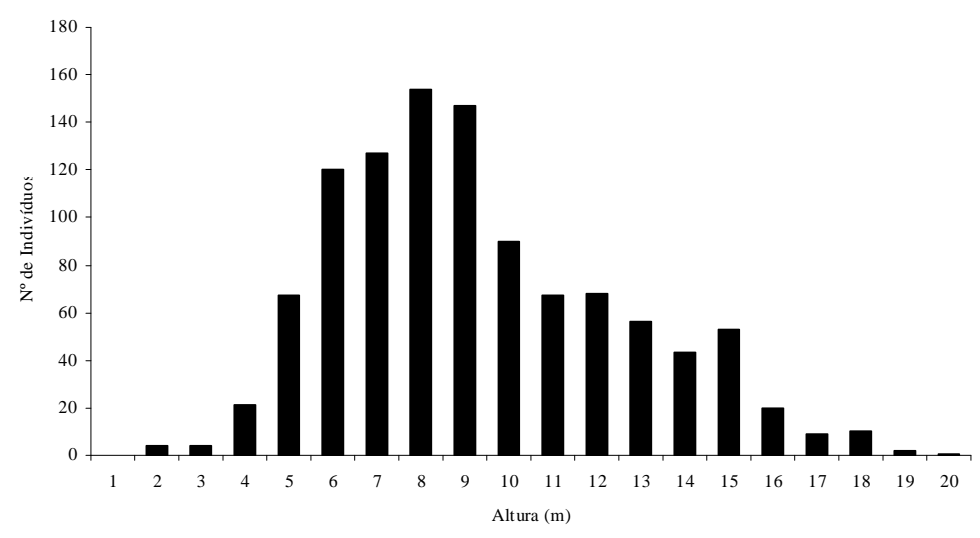

Figura 2. Distribuição dos indivíduos em classes de altura amostrados no levantamento estrutural em remanescente de floresta estacional no sul do Brasil.

Figure 2. Distribution of surveyed individuals by height classes in a remnant of seasonal forest in southern Brazil.

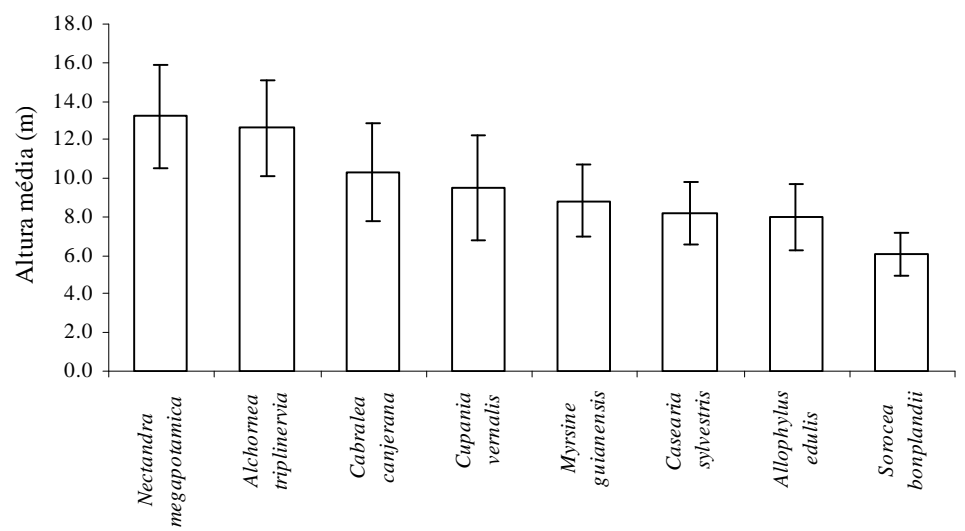

Figura 3. Altura média das espécies que apresentaram pelo menos 40 indivíduos amostrados no levantamento estrutural em remanescente de floresta estacional no sul do Brasil.

Figure 3. Average height from species which presented at least 40 individuals surveyed in the forest inventory in a remnant of seasonal forest in southern Brazil.

Alchornea triplinervia e Nectandra megapotamica apresentaram médias de altura similares (maiores que $12 \mathrm{~m}$ ), sendo superiores às demais espécies, e determinaram o andar superior da comunidade amostrada. Myrsine guianensis, Cabralea canjerana, Allophylus edulis, Cupania vernalis e Casearia sylvestris, com médias entre $8 \mathrm{~m}$ e $11 \mathrm{~m}$, ocuparam o estrato médio da comunidade. Por fim, Sorocea bonplandii ocupou o estrato inferior, com altura média de $6 \mathrm{~m}$ (Figura 3). Essas oito espécies, por apresentarem mais de 40 indivíduos no levantamento, ajudam a compor os três estratos de altura observados (sub-bosque, estrato médio e estrato superior). Esse fato pode ser atribuído à facilidade de adaptação dessas 
espécies a diversos ambientes, uma vez que todas possuem ampla distribuição no território sul-riograndense, sendo frequentemente encontradas em levantamentos florísticos e estruturais realizados em diversas regiões do estado (JARENKOW; WAECHTER, 2001; JURINITZ; JARENKOW, 2003; BUDKE et al., 2004; LINDENMAIER; BUDKE, 2006; GIEHL et al., 2007; DE MARCHI; JARENKOW, 2008).

Observou-se que a maior parte dos indivíduos amostrados no levantamento estrutural é de ampla distribuição (81\%), assim como a maior parte das espécies (51\%). Quanto à proporção de indivíduos, essas taxas também foram observadas em outros estudos na região, por Jarenkow; Waechter (2001), em uma floresta na região central do Rio Grande do Sul, e também por Budke et al. (2004), os quais estudaram uma floresta ribeirinha no município de Santa Maria. Entretanto, nesses trabalhos, a maior parte das espécies amostradas enquadrou-se no contingente das florestas estacionais (oeste). No estudo de Jarenkow (1994), o contingente oeste representou somente $4 \%$ dos indivíduos amostrados e $13 \%$ das espécies, enquanto que $57 \%$ dos indivíduos amostrados e $29 \%$ das espécies amostradas foram espécies de ampla distribuição.

No presente estudo, a riqueza específica do contingente oeste foi relativamente alta (42\%). Já o baixo número de indivíduos pertencentes a esse mesmo contingente (17\%) se contrapôs ao observado por Lindenmaier; Budke (2006) em uma floresta decídua no município de Cachoeira do Sul. No estudo de Jarenkow (1994), no município de Morrinhos do Sul, região leste da encosta da Serra Geral, 38\% dos indivíduos amostrados e 55\% das espécies pertenceram ao contingente Atlântico. No presente estudo, as espécies provenientes do contingente Atlântico (leste) apresentaram, além de um baixo número de indivíduos (2\%), também uma baixa riqueza (3\%), da mesma forma que os trabalhos de Budke et al. (2004) e Lindenmaier; Budke (2006). Já no estudo de Jarenkow; Waechter (2001), realizado na região central do Rio Grande do Sul, também foi constatado baixa riqueza de espécies provenientes do leste, porém boa parte do sub-bosque foi formado por espécies provenientes desse mesmo contingente, enquanto que o estrato superior foi ocupado por árvores emergentes, em sua maioria provenientes do contingente das florestas estacionais. No presente estudo, as espécies de ampla distribuição destacam-se pelo alto valor de riqueza e pelo grande número de indivíduos amostrados.

\section{CONCLUSÕES}

- Houve predomínio de espécies de ampla distribuição geográfica, associadas a um elevado número de indivíduos, ao contrário do que observado para os contingentes Atlântico e oeste, ambos com espécies de baixa densidade. Por outro lado, houve grande contribuição de espécies do contingente oeste, refletindo numa área de transição entre formações vegetacionais.

- Determinaram-se três estratos arbóreos, em que a maior parte do sub-bosque, do estrato médio e do estrato superior foi composta por indivíduos de ampla distribuição. Isso, aliado ao fato de que a maior parte da riqueza também foi composta por espécies de ampla distribuição, demonstra a importância dessas espécies em ocupar e formar os diferentes estratos da comunidade estudada.

- Apesar da área da reserva apresentar um elevado número de espécies autóctones e uma elevada riqueza de espécies ameaçadas, faz-se necessário o uso de técnicas de monitoramento das espécies exóticas, em especial as exóticas consideradas invasoras.

\section{AGRADECIMENTOS}

Nossos sinceros agradecimentos a todos que contribuíram para a realização deste trabalho, em especial aos amigos Alexandre Somavilla, Edson Fiedler de Abreu Júnior, Fabiane Noronha e Samuel Lopes de Oliveira, pelo apoio e parceria nas centenas de saídas a campo; a João André Jarenkow, pelas sugestões e incentivo; a Marcos Sobral, pelo auxílio na identificação dos táxons e incentivo; à Universidade de Santa Cruz do Sul, pelas bolsas PUIC e PROBEX concedidas ao primeiro autor.

\section{REFERÊNCIAS}

APG II. An update of the Angiosperm Phylogeny Group classification for the orders and families of flowering plants. Botanical Journal of the Linnean Society, London, v. 141, p. 399-436, 2003. 
BERGAMIN, R. S.; MONDIN, C. A. Composição florística e relações fitogeográficas do componente arbóreo de um fragmento florestal no município de Barra do Ribeiro, Rio Grande do Sul, Brasil. Pesquisas. Botânica, São Leopoldo, v. 57, p. 217-230, 2006.

BIODIVERSITAS. Lista da flora brasileira ameaçada de extinção. 2005. Disponível em <http://www.biodiversitas.org.br/floraBr/consulta_fim.asp>. Acesso em: 15/1/2009.

BRASIL. Ministério do Meio Ambiente. Portaria No 16, de 18 de Março 2009. Diário Oficial da União, seção 1, n. 53. 2009.

BEHLING, H. Investigations into the Late Pleistocene and Holocene history of vegetation and climate in Santa Catarina (S Brazil). Vegetation History and Archaeobotany, Berlin, v. 4, p. 127-152, 1995.

BEHLING, H. Late Quaternary vegetational and climatic changes in Brazil. Review of Palaeobotany and Palynology, Amsterdam, v. 99, p. 143-156, 1998.

BEHLING, H.; BAUERMANN, S. G.; NEVES, P. C. Holocene environmental changes from the São Francisco de Paula region, southern Brazil. Journal of South American Earth Sciences, Oxford, v. 14, p. 631-639, 2001.

BEHLING, H. South and Southeast Brazilian grasslands during Late Quaternary times: a synthesis. Palaeogeography Palaeoclimatology Palaeoecology, Amsterdam, v. 177, p. 19-27, 2002.

BEHLING, H.; PILLAR, V.; ORLÓCI, L.; BAUERMANN, S. G. Late Quaternary Araucaria forest, grassland (Campos), fire and climate dynamics, studied by high resolution pollen, charcoal and multivariate analysis of the Cambará do Sul core in southern Brazil. Palaeogeography Palaeoclimatology Palaeoecology, Amsterdam, v. 203, p. 277-297, 2004.

BEHLING, H.; PILLAR, V.; BAUERMANN, S. G. Late Quaternary grassland (Campos), gallery forest, fire and climate dynamics, studied by pollen, charcoal and multivariate analysis of the São Francisco de Assis core in western Rio Grande do Sul (southern Brazil). Review of Palaeobotany and Palynology, Amsterdam, v. 133, p. 235-248, 2005.

BUDKE, J. C.; GIEHL, E. L. H.; ATHAYDE, E.; ZÁCHIA, R. A. Florística e Fitossociologia do Componente Arbóreo de uma Floresta Ribeirinha, Arroio Passo das Tropas, Santa Maria, RS, Brasil. Acta Botanica Brasilica, São Paulo, v. 18, n. 3, p. 581-598, 2004.

DE MARCHI, T. C.; JARENKOW, J. A. Estrutura do componente arbóreo de mata ribeirinha no rio Camaquã, município de Cristal, Rio Grande do Sul, Brasil. Iheringia. Série Botânica, Porto Alegre, v. 63, n. 2, p. 241-248, 2008.

EMPRESA BRASILEIRA DE PESQUISA AGROPECUÁRIA. Centro Nacional de Pesquisas de Solos (EMBRAPA-CNPS). Sistema brasileiro de classificação de solos. Brasília,DF, 1999. 412 p.

FILGUEIRAS, T. S.; BROCHADO, A. L.; NOGUEIRA, P. E.; GUALA, G. F. Caminhamento - Um método expedito para levantamentos florísticos qualitativos. Caderno de Geociências, Brasília, DF, v. 12, p. 39-43, 1994.

GIEHL, E. L. H.; ATHAYDE, E. A.; BUDKE, J. C.; GESING, J. P. A.; EISINGER, S. M.; CANTODOROW, T. S. Espectro e distribuição vertical das estratégias de dispersão de diásporos do componente arbóreo em uma Floresta Estacional no sul do Brasil. Acta Botanica Brasilica, São Paulo, v. 21, p. 137-145, 2007.

INTERNATIONAL UNION FOR CONSERVATION OF NATURE AND NATURAL RESOURCES (IUCN). IUCN guidelines for the prevention of biodiversity loss caused by alien invasive species. 51st meeting of Council, 2000.

INTERNATIONAL UNION FOR CONSERVATION OF NATURE AND NATURAL RESOURCES (IUCN). Red list of threatened species. 2008. Disponível em: <http://www.biodiversitas.org.br/floraBr/iucn.pdf>. Acesso em: 8/4/2009. 
JARENKOW, J. A. Estudo fitossociológico comparativo entre duas áreas com mata de encosta no Rio Grande do Sul. 125 p. Tese (Doutorado) - Centro de Ciências e da Saúde, Universidade Federal de São Carlos, São Carlos, 1994.

JARENKOW, J. A.; WAECHTER, J. L. Composição, estrutura e relações florísticas do componente arbóreo de uma floresta estacional no Rio Grande do Sul, Brasil. Revista Brasileira de Botânica, São Paulo, v. 24, n. 3, p. 263-272, 2001.

JURINITZ, C. F.; JARENKOW, J. A. Estrutura do componente arbóreo de uma floresta estacional na Serra do Sudeste, Rio Grande do Sul, Brasil. Revista Brasileira de Botânica, São Paulo, v. 26, n. 4, p. 475-487, 2003.

KERN, A. A. Paleopaisagens e o povoamento pré-histórico do Rio Grande do Sul. In: KERN, A. A. (Org.). Arqueologia pré-histórica do Rio Grande do Sul. Porto Alegre: Mercado Aberto, 1991.

LINDENMAIER, D. S.; BUDKE, J. C. Florística, diversidade e distribuição espacial das espécies arbóreas em uma floresta estacional na bacia do rio Jacuí, sul do Brasil. Pesquisas. Botânica, São Leopoldo, v. 57, p. 193-216, 2006.

MACK, R. N.; CHAIR; SIMBERLOFF, D.; LONSDALE, W. M.; EVANS, H.; CLOUT, M.; BAZZAZ, F. Biotic invasions: causes, epidemiology, global consequences and control. Issues in Ecology, Ithaca,n. 5, Spring. 2000.

MALUF, J. R. T. Nova classificação climática do Rio Grande do Sul. Revista Brasileira de Agrometeorologia, Santa Maria, v. 8, n. 1, p. 141-150, 2000.

MULLER-DOMBOIS, D.; ELLENBERG, H. Aims and methods of vegetation ecology. New York: J. Wiley. 1974. 542 p.

PARANÁ. Secretaria do Meio Ambiente e Recursos Hídricos. Instituto Ambiental do Paraná. Portaria IAP n ${ }^{\circ} 095$ de 22 de Maio de 2007. Reconhece a Lista Oficial de Espécies Exóticas Invasoras para o Estado do Paraná, estabelece normas de controle e dá outras providências.

RAMBO, B. O elemento andino no pinhal riograndense. Anais Botânicos do Herbário Barbosa Rodrigues, Itajaí, v. 3, n. 3, p. 7-39, 1951.

RAMBO, B. História da flora do planalto riograndense. Anais Botânicos do Herbário Barbosa Rodrigues, Itajaí, v. 5, p. 185-232, 1953.

RAMBO, B. A fisionomia do Rio Grande do Sul. 2. ed. Porto Alegre: Selbach, 1956. 471 p.

RAMBO, B. Migration routes of the south brazilian rain forest. Pesquisas. Botânica, São Leopoldo, v. 12, p. 1-54. 1961.

RIO GRANDE DO SUL. Decreto Estadual n. 42.099 de 1/1/2003. Dispõe sobre a lista final das espécies da flora ameaçadas - RS. Publicado em:

SMITH, A. R.; PRYER, K. M.; SCHUETTPELZ, E.; KORALL, P.; SCHNEIDER, H.; WOLF, P. G. A classification for extant ferns. Taxon, Wien, v. 55, n. 3 p. 705-731, 2006.

SOBRAL, M.; JARENKOW, J. A.; BRACK, P.; IRGANG, B.; LAROCCA, J.; RODRIGUES, R. S. Flora arbórea e arborescente do Rio Grande do Sul. Porto Alegre: Rima-NovoAmbiente, 2006. 350 p.

STRECK, E. V.; KÄMPF, N.; DALMOLIN, R. S. D.; KLAMT, E.; NASCIMENTO, P. C.; SCHNEIDER, P. Solos do Rio Grande do Sul. Porto Alegre: EMATER/UFRGS, 2002. 107 p.

WAECHTER, J. L. Padrões geográficos na flora atual do Rio Grande do Sul. Ciência \& Ambiente, Santa Maria, v. 24, n. 24, p. 93-108, 2002.

ZILLER, S. R.; GALVÃO, F. O processo de degradação da Estepe Gramíneo-Lenhosa no Paraná por contaminação biológica de Pinus elliotti e P. taeda. Floresta, Curitiba, 2003. 